\title{
Shining the Light on the MotionWatch8 Light Sensor for Sleep and Aging Research: What Can We Measure and What Are We
}

\section{Missing?}

\author{
Ryan S. Falck ${ }^{\mathrm{a}, \mathrm{b}}$, Rachel A. Crockett ${ }^{\mathrm{a}, \mathrm{b}}$, Jennifer C. Davis ${ }^{\mathrm{a}, \mathrm{b}, \mathrm{c}}$, Karim M. Khan ${ }^{\mathrm{b}, \mathrm{d}}$ \\ and Teresa Liu-Ambrose $\mathrm{a}^{\mathrm{a}, \mathrm{b}, *}$ \\ ${ }^{a}$ Aging, Mobility, and Cognitive Neuroscience Laboratory, Department of Physical Therapy, University of British \\ Columbia, Vancouver, BC, Canada \\ ${ }^{\mathrm{b}}$ Centre for Hip Health and Mobility, University of British Columbia, Vancouver, BC, Canada \\ ${ }^{\mathrm{c}}$ Social \& Economic Change Laboratory, Faculty of Management, University of British Columbia-Okanagan \\ Campus, Kelowna, BC, Canada \\ ${ }^{\mathrm{d}}$ Department of Family Practice, Faculty of Medicine, University of British Columbia, Vancouver, BC, Canada
}

Accepted 18 December 2020

Pre-press 11 January 2021

\begin{abstract}
.
Background: Poor sleep is common among older adults at risk for dementia and may be due to circadian dysregulation. Light is the most important external stimulus to the circadian clock and bright light therapy (BLT) has been used for $>20$ years to help realign circadian rhythms. However, the ability of field methods (e.g., actigraphy) to accurately determine the type and intensity of light is unknown.

Objective: We examined the ability of the MotionWatch8 (MW8) light sensor to determine: 1) light versus dark, 2) electrical light versus daylight, and 3) device-based BLT versus light which was not BLT.

Methods: We tested the MW8 under 17 daily light scenarios. Light exposure data was collected for 5 minutes during each scenario. Concurrently, we measured light exposure using the LT40 Light Meter, a sensitive measure of light intensity. We then developed individual cut-points using receiver operator characteristics analyses to determine optimal MW8 cut-points for 1) light versus dark; 2) electrical light versus daylight; and 3) light from a BLT box versus light which was not BLT. Bland-Altman plots tested the precision of the MW8 compared to the LT40.

Results: The MW8 accurately discriminated light versus dark ( $>32$ lux), and electrical light versus daylight ( $<323$ lux). However, the MW8 had poor accuracy for 1) discriminating BLT from light which was not BLT; and 2) low precision compared to the LT40.

Conclusion: The MW8 appears to be able to discern light versus dark and electrical light versus daylight; however, there remains a need for accurate field methods capable of measuring light exposure.
\end{abstract}

Keywords: Actigraphy, light, luxometer, measurement, sleep

\footnotetext{
*Correspondence to: Dr. Teresa Liu-Ambrose, University of British Columbia, Department of Physical Therapy, Faculty of Medicine, Aging, Mobility, and Cognitive Neuroscience Lab, Vancouver Coastal Health Research Institute, 212 - 2177 Wesbrook Mall, Vancouver, BC V6T 1Z3, Canada. E-mail: teresa.ambrose@ubc.ca.
}

\section{INTRODUCTION}

Poor sleep is a risk factor for cognitive decline and dementia [1]. Older adults with mild cognitive impairment (MCI), an early transition stage from healthy cognition to dementia [2], experience poorer 
sleep than their healthy peers [3]. Promoting better sleep among older adults with MCI is therefore critical. Light, both exposure to daylight and electrical 'bright light therapy' (BLT), may be one way to ameliorate the consequences of poor sleep on cognitive health $[4,5]$.

Sleep is closely tied to circadian rhythms [6], the approximately 24-hour biological clock that helps regulate the sleep-wake cycle $[7,8]$. The endogenous human biological clock is synchronized with the solar light-dark cycle through external stimuli (i.e., zeitgebers) by a process known as entrainment [4]. Circadian alignment is critical for good quality sleep and MCI is associated with circadian dysregulation such as a greater number of sleep disruptions, and decreased amplitude and robustness of circadian sleep-wake rhythms [9].

The principal entraining factor of the human biological clock is light, which exerts its influence on light-sensitive receptors in the retina [10]. Photoreceptive melanopsin expressing ganglion cells in the retina (ipRGCs) are at the center of circadian regulation, conveying and receiving input to and from conventional visual photoreceptors (i.e., rods and cones) $[11,12]$. One important target of ipRGCs is the suprachiasmatic nuclei (SCN), the "master circadian pacemaker" of the brain [13]. However, human aging is associated with declines in light sensitivity due to lens-yellowing and senescent miosis $[14,15]$. Alzheimer's disease is also associated with a loss of ipRGCs and the SCN may also becomes less sensitive to signals from the retina compared with normal aging $[16,17]$. These underlying changes in the structures which control circadian regulation likely explain the prevalence of sleep disruptions in dementia [18], and thus it is critical to determine therapeutic strategies which will help maintain or realign circadian rhythms in older adults with cognitive impairment.

BLT is an increasingly popular strategy for promoting circadian alignment and sleep among older adults with cognitive impairment $[9,19]$. Researchers have speculated that effectively timed BLT may strengthen the entrainment of the biological clock to the solar light-dark cycle, increase the amplitude of the circadian rhythm, and improve alertness and reduce daytime sleepiness, all of which might help promote better sleep at night [6]. The efficacy of BLT as a therapy to promote sleep and cognitive health in people with cognitive impairment is currently inconclusive $[5,20]$. One possible explanation is that the biological clock is not equally amenable to shifts at each phase in the circadian rhythm [21], such that BLT may require proper timing which is based on an individual's circadian rhythm [9]. Accurately measuring participants' light exposure before, during, and after BLT may help elucidate how to maximize treatment efficacy.

However, the measurement of light is not straightforward. Price and colleagues [22] recently examined 11 different light-recording devices (i.e., dosimeters and/or light-logging actigraphs). The authors determined that optical performance varied considerably between devices, likely due to differences in sensor position and insufficient linear ranges (i.e., $<200,000$ lux) of devices; the authors suggested that researchers should also consider other variables when choosing a device such as cost, availability, reliability, specification risk, and actigraphy performance. While there are guidelines for the measurement of light [23, 24], measuring individual light exposure over time, and in a field setting, is still difficult for four reasons. First, the current methods for longitudinally measuring individual exposure to light is restricted to wearable light meters [25]. The primary indices for many of these devices is not light; many of these devices are designed to measure sleep, circadian rhythms, or both. Second, although different types of light can differentially affect circadian biology, most wearable light meters cannot easily estimate the different components of light [26], and it may require multiple sensors to be worn in order to obtain highly accurate light estimates relative to retinal light exposure [27]. Third, wearable light meters still lack a readilyavailable technique to make simple discriminations between types of light which an older adult may be exposed to on a daily basis, such as: light versus dark, electrical light versus daylight, or device-based BLT versus any light exposure which occurs while not using the BLT box. Past studies examining how light impacts sleep and circadian rhythms have made simple cut-offs for discerning daylight from other light (>1000 lux is assumed to be daylight) $[28,29]$; however, this method for determining daylight from other light is not based on formal evidence. These differences in the type and amount of light to which older adults are exposed could have important implications for their sleep and circadian physiology [20]. Fourth, it is still unclear how accurate these wearable light meters are for measuring light under different every day conditions (e.g., different clothing, different times of day, etc.). The lack of field measures which can accurately measure light limits our ability to understand how light impacts circadian regulation [30]. 
One promising field-method which can estimate light exposure, and is commonly used in sleep research is actigraphy. The device is generally worn on the wrist to record movement, and data are collected for display and analysis of activity/inactivity which in turn can be further analyzed to estimate wake versus sleep [31]; circadian parameters can also be indexed based on movement over the 24hour period [32]. Most actigraphs are also equipped with a light sensor with a range of lux, although only one study (to our knowledge) has used actigraphs to examine the impacts of light exposure on circadian regulation in older adults with cognitive impairment. Ancoli-Isreal [29] and colleagues found that older adults with dementia had poorer circadian regulation and less bright light exposure than older adults without dementia; research using dosimeters have found similar results [33], suggesting that actigraphy is an under-utilized tool for examining how light can impact older adult circadian regulation and cognitive health.

Standard research protocols for estimating sleep using actigraphy include light exposure data as a parameter for scoring [34]; however, we found no reports of either (i) the accuracy of these devices in typical daily settings compared to a light emitting diode (LED) light meter, or (ii) whether actigraphs can discriminate different types of light exposure (e.g., electrical light versus daylight). Hence, we conducted a measurement study to determine 1) the ability of the MotionWatch8 (MW8; CamNtech) light sensor to discern different types of light exposure; and 2) the accuracy of the MW8 compared to a LED light meter.

\section{MATERIALS AND METHODS}

This measurement study was conducted between June $16^{\text {th }}, 2018$ and January $11^{\text {th }}, 2019$. All data were collected by two authors (RSF and RAC).

\section{Instruments and software}

We used the MW8 to collect light data (i.e., lux). The MW8 is a wrist-worn actigraph which has a lux range from 0 to 64000 lux and samples the light exposure (average lux) at a frequency of $1 \mathrm{~Hz}$. In keeping with current guidelines, we used 60 second epochs for MW8 data collection [34]. We used the MotionWare software to download and process the data to an excel spreadsheet which provided epoch-by-epoch lux measurements.

\section{Procedure}

We used four MW8 devices to measure light in 17 different light scenarios-representing the light an individual's retina is exposed to at various times of the day for common daily indoors and outdoors activities (Table 1). The order of use for each MW8 was randomly assigned for each light scenario. Each scenario was tested on each watch for a period of five minutes. To adjust for seasonal and weather differences in sunlight, we conducted all outdoor measurements on two separate days (i.e., June 16, 2018 and January 11,2019$)$ at approximately the same time of day.

For each light scenario, the event marker time stamp was pressed at the beginning and end of each MW8 recording. All instruments were synchronized, and time was recorded at the beginning and end of each activity to ensure appropriate data comparisons were made across all devices. Using a stopwatch, light intensity was recorded each minute using the LT40 LED Light Meter (Extech Instruments; USA). The LT40 is a calibrated light meter which has a range of 0 to 400,000 lux with a margin of error of $\pm 3 \%$. Each recording from the LT40 was taken at approximately the same height as the MW8 being tested, and at $\sim 5 \mathrm{~cm}$ adjacent to the MW8 being tested. The LT40 was held horizontally and parallel to the ground throughout the experiment.

With the exception of the dim light condition, we performed each condition twice (five minutes each): once where the research assistant wearing the MW8 was in short sleeves (i.e., the MW8 was uncovered), and again wearing long sleeves (i.e., the MW8 was covered by a shirt sleeve). We did this to account for differences in seasonal attire. We conducted two separate trials of each light scenario for each MW8 device - a total of 680 epochs recorded. Each measurement for the MW8 and LT40 were recorded on an epoch-by-epoch basis in an excel spreadsheet.

\section{Statistical analyses}

All analyses were conducted in $\mathrm{R}$ version 3.5.1 (Supplementary Material 1). Paired $t$-tests examined differences in lux for each condition based on whether the MW8 was worn with sleeves or no sleeves, and differences in lux between the MW8 and the LT40. Bland-Altman plots compared the estimated MW8 lux to the estimated LT40 lux [35].

We then developed individual cut-points using receiver operator characteristics (ROC) analyses to 
Table 1

Description of the 17 light scenarios used

\begin{tabular}{lcc}
\hline Condition & Long Sleeves & Short Sleeves \\
\hline Morning sunlight (approximately 10:30 AM) outdoors & $\checkmark$ & $\checkmark$ \\
Indoor electrical light & $\checkmark$ & $\checkmark$ \\
Indoor electrical light sitting 1 meter from a commercially & $\checkmark$ & $\checkmark$ \\
available BLT device (Philips goLITE BLU) & $\checkmark$ & $\checkmark$ \\
Mid-day sunlight outdoors (i.e., 1:00 PM) & $\checkmark$ & $\checkmark$ \\
Indoor electrical light sitting $\sim 1$ meter from a computer screen & $\mathbf{X}$ \\
Room with dim electrical light (i.e., only enough to read the LT40 & $\checkmark$ & $\checkmark$ \\
LED Light Meter) & $\checkmark$ & $\checkmark$ \\
Indoor electrical light while using a smart phone $\sim 30$ cm from face & & $\checkmark$ \\
Room with dim electrical light (i.e., only enough to read the LT40 & $\checkmark$ & $\checkmark$ \\
LED Light Meter) while using a smartphone & $\checkmark$ & \\
Evening sunlight (i.e., $~ 7: 00$ PM) outdoors & & \\
\hline
\end{tabular}

Each condition was performed for a period of five minutes. $\checkmark=$ Condition performed; $\mathbf{X}=$ Condition not performed.

Table 2

Description of how each light scenario was classified for each of the receiver operating curve analyses

\begin{tabular}{lccc}
\hline Condition & $\begin{array}{c}\text { Light versus } \\
\text { Dark }\end{array}$ & $\begin{array}{c}\text { Indoor versus } \\
\text { Outdoor }\end{array}$ & $\begin{array}{c}\text { BLT versus } \\
\text { Other }\end{array}$ \\
\hline Morning Sunlight & Light & Outdoor & Other \\
Indoor Electrical Light & Light & Indoor & Other \\
Bright Light Box $^{1}$ & Light & Indoor & BLT \\
Mid-day sunlight $^{\text {Computer Screen }}{ }^{2}$ & Light & Outdoor & Other \\
Dim Light $^{3}$ & Light & Indoor & Other \\
Smart Phone $^{4}$ & Dark & Indoor & Other \\
Dim Light while Using a Smart Phone $^{5}$ & Light & Indoor & Other \\
Evening Sunlight $^{\text {Sving }}$ & Dark & Indoor & Other \\
\hline
\end{tabular}

${ }^{1}$ Indoor electrical light sitting $\sim 1$ meter from a commercially available BLT device (Philips goLITE BLU).

${ }^{2}$ Indoor electrical light sitting $\sim 1$ meter from a computer screen. ${ }^{3}$ Room with dim electrical light (i.e., only enough to read the LT40 LED Light Meter); no sleeves condition used, given low light exposure. ${ }^{4}$ Indoor electrical light while using a smart phone $\sim 30 \mathrm{~cm}$ from RAC's face. ${ }^{5}$ Room with dim electrical light (i.e., only enough to read the LT40 LED Light Meter) while using a smartphone $\sim 30 \mathrm{~cm}$ from face.

determine optimal MW8 cut-points for 1) light versus dark; 2) electrical light versus daylight; and 3) light from a BLT box versus any light exposure which occurred while not using the BLT box (i.e., no BLT). We used the known position of the MW8 as the reference standard. For each ROC curve, light exposure type was coded as either 0 or 1 according to the cut-point which was being established. For example, for determining electrical light versus daylight, a "1" was assigned to all minutes when the MW8 was being exposed to sunlight outdoors, and a " 0 " was assigned to all minutes when the MW8 was not being exposed to sunlight. A complete description of which scenarios were assigned for each ROC analysis is described in Table 2. Cut-points were established for each type of light exposure using the Youden index on the ROC curve [36]. We determined the sensitivity (e.g., for light versus dark, accurately determined light when the condition was light) and specificity (for light versus dark, accurately determined it was not light when the condition was not light).

\section{RESULTS}

Differences in lux by condition and by device are described in Table 3. With the exception of the morning light condition, wearing sleeves was associated with the MW8 recording lower lux, as compared with not wearing sleeves. There were also significant differences in lux between the MW8 when sleeves were worn and the LT40 for all conditions $(p<0.001)$; the MW8 was associated with lower lux for each condition. When participants did not wear sleeves, the MW8 was associated with significantly lower lux compared to the LT40 for seven of the nine conditions $(p<0.05)$. Figure 1 illustrates the differences in lux between the MW8 and LT40. The 
Table 3

Differences in lux between the MotionWatch8 and LT40 LED Light Meter (680 total epochs), as a function of wearing long shirt sleeves

\begin{tabular}{|c|c|c|c|c|c|c|c|c|}
\hline \multirow[b]{2}{*}{ Condition } & \multicolumn{3}{|c|}{ MotionWatch8 Lux } & \multirow{2}{*}{$\begin{array}{l}\text { LT40 LED } \\
\text { Light Meter } \\
\text { Lux }\end{array}$} & \multicolumn{4}{|c|}{$\begin{array}{l}\text { Between Instrument Differences } \\
\text { (MotionWatch8 - LT40) }\end{array}$} \\
\hline & Sleeves & No Sleeves & $p$ & & Sleeves & $p$ & No Sleeves & $p$ \\
\hline Morning Sunlight & $24803(25004)$ & $18671(10568)$ & 0.159 & $87992(29051)$ & -63601 & $<0.001$ & -68908 & $<0.001$ \\
\hline Indoor Electrical Light & $59(22)$ & $84(30)$ & $<0.001$ & $88(11)$ & -29 & $<0.001$ & -4 & 0.243 \\
\hline Bright Light Box ${ }^{1}$ & $70(40)$ & $138(32)$ & $<0.001$ & $116(12)$ & -41 & $<0.001$ & 18 & 0.002 \\
\hline Mid-day Sunlight & $38330(41917)$ & $86720(44256)$ & $<0.001$ & $102700(16690)$ & -66376 & $<0.001$ & -13974 & 0.067 \\
\hline Computer Screen ${ }^{2}$ & $78(65)$ & $146(40)$ & $<0.001$ & $134(10)$ & -60 & $<0.001$ & 16 & 0.011 \\
\hline $\operatorname{Dim}_{\text {Light }}{ }^{3}$ & - & $11(6)$ & - & $1(1)$ & - & - & 11 & $<0.001$ \\
\hline Smart Phone ${ }^{4}$ & $53(35)$ & $75(30)$ & 0.003 & $90(13)$ & -36 & $<0.001$ & -16 & 0.002 \\
\hline $\begin{array}{l}\text { Dim Light while Using a } \\
\text { Smart Phone }\end{array}$ & $14(10)$ & $83(96)$ & $<0.001$ & $58(105)$ & 13 & $<0.001$ & -33 & 0.015 \\
\hline Evening Sunlight & $96(206)$ & $468(749)$ & 0.004 & $346(307)$ & -128 & $<0.001$ & 1 & 0.991 \\
\hline
\end{tabular}

${ }^{1}$ Indoor electrical light sitting $\sim 1$ meter from a commercially available BLT device (Philips goLITE BLU). ${ }^{2}$ Indoor electrical light sitting $\sim 1$ meter from a computer screen. ${ }^{3}$ Room with dim electrical light (i.e., only enough to read the LT40 LED Light Meter); no sleeves condition used, given low light exposure. ${ }^{4}$ Indoor electrical light while using a smart phone $\sim 30 \mathrm{~cm}$ from RAC's face. ${ }^{5}$ Room with dim electrical light (i.e., only enough to read the LT40 LED Light Meter) while using a smartphone $\sim 30 \mathrm{~cm}$ from face.

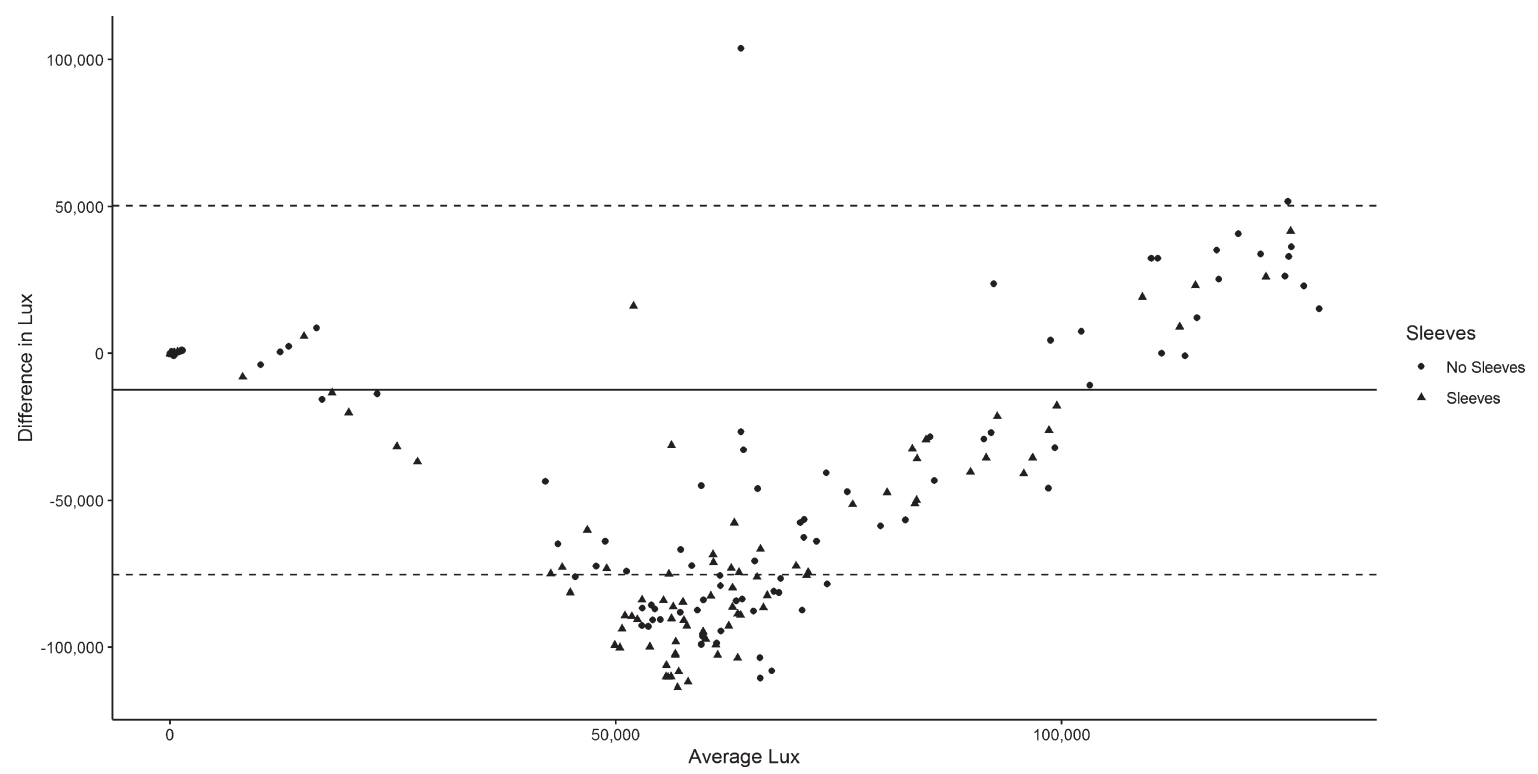

Fig. 1. Bland Altman plot for determining the precision of the MotionWatch8 (MW8) light sensor versus the LT40 LED Light Meter at different intensities of light. The solid line is the mean difference in lux between the MW8 and LT40. Dashed lines are the upper and lower limits of agreement for differences in lux readings between the devices.

MW8 tended to underestimate lux compared to the LT40 - particularly when sleeves were worn and also when average lux was $\sim 50,000-100,000$.

The area under the curve (AUC) for light versus dark (AUC $=0.843 ; 95 \%$ CI: $[0.807,0.880])$, as well as electrical light versus daylight $(\mathrm{AUC}=0.812$; 95\% CI: [0.769, 0.855]) showed good accuracy for distinguishing between conditions (Fig. 2). The cut-point of distinguishing light versus dark using the MW8 was $>32$ lux (Sensitivity $=84.11 \%$; Specificity $=83.33 \%$ ), and the cut-point for determining electrical light versus daylight was $>323$ $\operatorname{lux}($ Sensitivity $=100 \%$; Specificity $=71.25 \%)$. However, the AUC for distinguishing BLT box use versus no BLT (AUC $=0.514 ; 95 \%$ CI: $[0.466,0.563]$ ) showed poor accuracy [36]. Given the low accuracy for each of these ROCs, we did not determine cutpoints for this diagnostic.

\section{DISCUSSION}

Over 350 years ago, Galileo Galilei implored scientists to "measure what is measurable, and make 

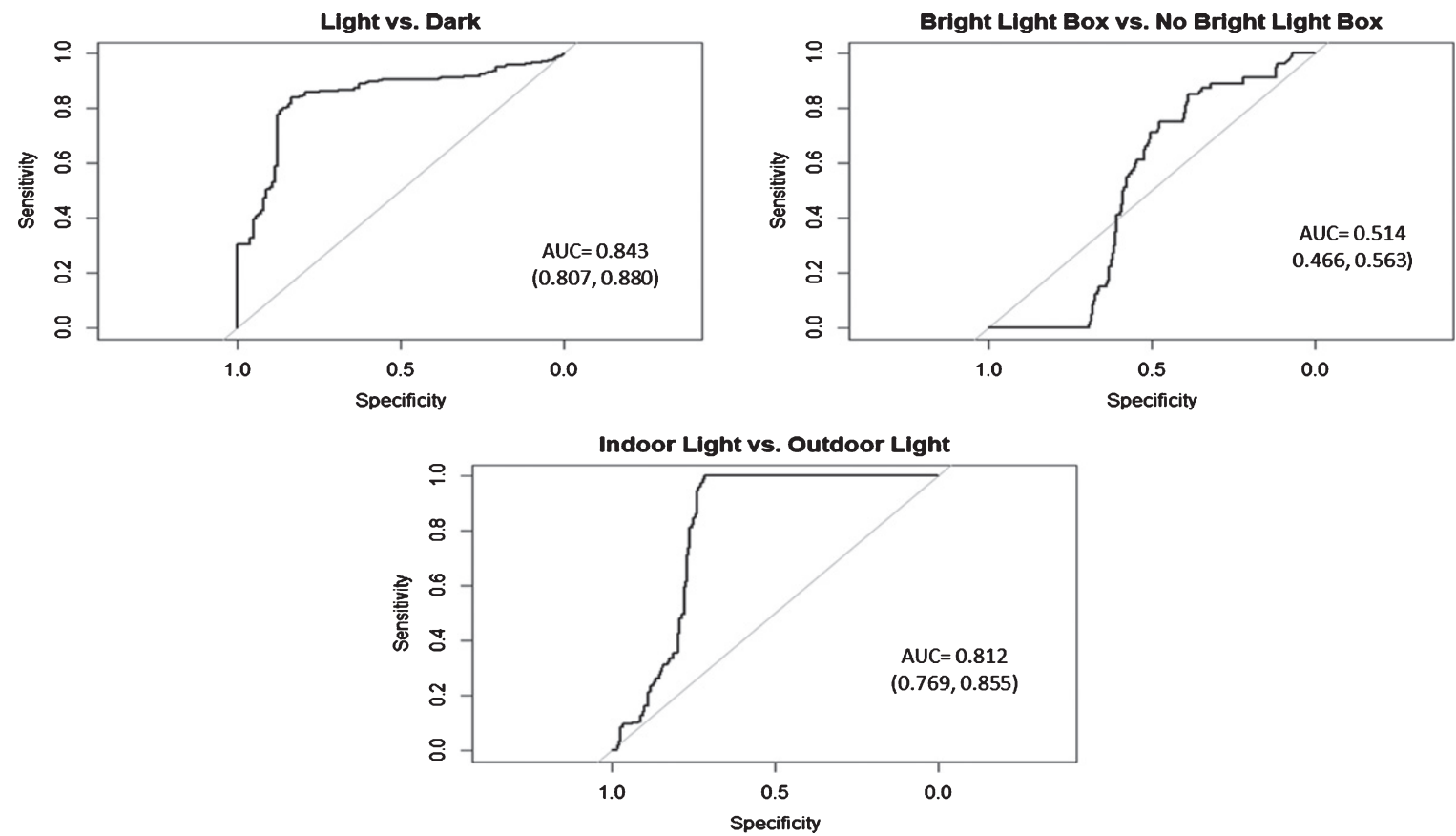

Fig. 2. Receiver operating characteristic (ROC) curves for determining the diagnostic accuracy of the MotionWatch8 light sensor for discriminating 1) light versus dark; 2) light from a bright light therapy (i.e., blue light) box versus other types of light; and 3) electrical versus daylight. AUC, area under the curve.

measurable what is not so." It is perhaps a little bit ironic that Galileo's own attempts to measure the principles of light, in particular, the speed of light, were inconclusive. As for our own experiment, we aimed to help sleep and circadian rhythm researchers who use field methods measure light more accurately - to document the knowns and the unknowns of the MW8 light sensor. Our results indicate the MW8 can accurately discriminate light versus dark, and electrical light versus daylight. However, the device is not sensitive enough to be used as a measure of adherence to a BLT program.

The reliability of the MW8 light sensor is also modest. We determined there were substantial differences in lux depending on whether or not the study participant is wearing long sleeves or not. This may seem self-evident, but the standard guidelines for using the MW8 make no mention of shirt/sweater/coat choices. Given that we also found substantial discrepancies in lux between the MW8 light sensor and our criterion measure, the LT40, this suggest that the MW8 does not provide reliable or accurate estimates of lux.

Past studies examining how light impacts sleep and circadian rhythms have made simple cut-offs for discerning daylight from electrical light (>1000 lux is assumed to be daylight) [28, 29]; however, this method was not based on formal evidence. Our results suggest that the MW8 has a lower cut-off for determining daylight from electrical light (i.e., >323 lux is daylight). This low cut-off was likely caused by low indoor light levels within the building in which we tested the device, and thus it is possible that more illuminated buildings would incorrectly classify a person as outside. Given that a cut-off of $>1000$ lux still provides similar sensitivity $(100 \%)$ and specificity $(69.17 \%)$ to a cut-off of $>323$ lux, we suggest that $>1000$ lux be used as the cut-off for determining indoor versus outdoor.

Our data suggest that the MW8 light sensor should be limited to determining light versus dark (or dim light), and electrical light versus daylight. Our cutpoints for determining light versus dark ( $>32$ lux) are well above the threshold of civil twilight ( $\sim 3.3$ lux $)$, the period after sunset or before sunrise when there is still enough light for ordinary outdoor occupations [37]. This suggests a highly conservative estimate for discriminating light versus dark. Light exposure data is already used as part of sleep scoring methods for actigraphy [34]; however, light exposure is often considered secondary to sleep diary information which is used to calibrate sleep window onset (i.e., "lights out") and sleep window conclusion ("got up"). Even 
though our cut-point for light versus dark is quite high, the cut-point has good sensitivity and specificity [36]. Hence, our a priori criterion for determining whether lights are "on" or "off" will also streamline and automate the process of using actigraphy for sleep analyses.

We also think using a cut-point of $>1000$ lux for determining electrical light versus daylight might be useful for researchers interested in examining how time spent outdoors versus indoors can impact sleep and circadian rhythms. Some evidence has suggested that greater amounts of outdoor sunlight can promote better sleep and circadian regulation among older adults with MCI or dementia [38]. Our study thus provides a simple metric for researchers to quantify the amount of time that older adults spend outdoors.

Nonetheless, the accuracy of the MW8 for determining the exact intensity of light exposure appears to be limited. There appears to be substantial variability in the accuracy of the MW8 sensor compared with the LT40 at different light intensities. The use of lux as an outcome variable for the MW8 should thus be treated cautiously, and we instead suggest using our cut-points to capture different aspects of light exposure.

\section{Study limitations}

Our study is limited to the MW8, and other actigraphs may have different accuracies and diagnostic capabilities. We did not examine the test-retest reliability of the MW8 light sensor. While we used 17 different light scenarios, the scenarios we chose were sedentary activities wherein the MW8 was more or less stable throughout. Unfortunately, we cannot generalize our findings and our data to different contexts beyond these light scenarios. We also did not examine sleep related activities (i.e., lying in bed, etc.). We collected outdoor data on days of temperate weather, and thus light exposure data might be different on days of inclement weather. Our results might also be specific to the location where data were collected (i.e., Vancouver, Canada).

Color temperature may also play an important role in the body's circadian rhythm [39]. Short high-energy light waves (e.g., blue and white light) suppress melatonin and encourages wakefulness [21]. However, we did not examine whether color temperature impacted illuminance accuracy of the MW8.

There may also be discrepancies between light measured at the wrist and light measured at the retina.
While we assumed that the MW8 provides an estimation of retinal light exposure, the device is worn on the wrist. There are several devices which are designed to specifically measure optical radiation $[27,40]$, and may more accurately reflect light exposure at the retina. Although the LT40 is a calibrated light meter for estimating light intensity, it still has a margin of error of $\pm 3 \%$. Finally, we categorized outdoor light as "other light" in our ROC analysis of BLT vs. other types of light, which may account for the low AUC. While this is possible, we do not think this is likely given the low average illuminance of BLT $(\sim 100$ lux $)$ which was roughly the same as most of the indoor light scenarios which we conducted.

\section{CONCLUSION}

In conclusion, our results suggest the MW8 light sensor can accurately discriminate between light and dark, and electrical light versus daylight. However, the MW8 lacks the accuracy to be used as a potential adherence measure for BLT interventions. As in every field of science, valid and accurate data are critical. We highlight the need for better instruments, capable of determining light intensity and type, in order for sleep research to advance.

\section{ACKNOWLEDGMENTS}

RAC is funded by the University of British Columbia Rehabilitation Sciences Scholarship. TLA is a Canada Research Chair (Tier II) in Physical Activity, Mobility, and Cognitive Neuroscience. KMK is the scientific director of the Canadian Institutes of Health Research Institute of Musculoskeletal Health and Arthritis (CIHR-IMHA). Funding for this work was provided by the Jack Brown and Family Alzheimer's Research Institute and Canada Research Chair Program. The sponsor had no role in the design, methods, data collections, analysis or preparation of this paper.

\section{CONFLICT OF INTEREST}

The authors have no conflict of interest to report.

\section{SUPPLEMENTARY MATERIAL}

The supplementary material is available in the electronic version of this article: https://dx.doi.org/ 10.3233/JAD-200242. 


\section{REFERENCES}

[1] Yaffe K, Falvey CM, Hoang T (2014) Connections between sleep and cognition in older adults. Lancet Neurol 13, 10171028.

[2] Petersen RC (2004) Mild cognitive impairment as a diagnostic entity. J Intern Med 256, 183-194.

[3] Naismith SL, Hickie IB, Terpening Z, Rajaratnam SW, Hodges JR, Bolitho S, Rogers NL, Lewis SJ (2014) Circadian misalignment and sleep disruption in mild cognitive impairment. J Alzheimers Dis 38, 857-866.

[4] Czeisler CA, Duffy JF, Shanahan TL, Brown EN, Mitchell JF, Rimmer DW, Ronda JM, Silva EJ, Allan JS, Emens JS (1999) Stability, precision, and near-24-hour period of the human circadian pacemaker. Science 284, 2177-2181.

[5] Roccaro I, Smirni D (2020) Fiat lux: The light became therapy. An overview on the bright light therapy in Alzheimer's disease sleep disorders. J Alzheimers Dis 77, 113-125.

[6] Borbély AA (1982) A two process model of sleep regulation. Hum Neurobiol 1, 195-204.

[7] Golombek DA, Rosenstein RE (2010) Physiology of circadian entrainment. Physiol Rev 90, 1063-1102.

[8] Czeisler CA, Gooley J (2007) Sleep and circadian rhythms in humans. In Cold Spring Harbor Symposia on Quantitative Biology, Cold Spring Harbor Laboratory Press, 72, pp. 579-597.

[9] Landry GJ, Liu-Ambrose T (2014) Buying time: A rationale for examining the use of circadian rhythm and sleep interventions to delay progression of mild cognitive impairment to Alzheimer's disease. Front Aging Neurosci 6, 325.

[10] Sharma VK, Chandrashekaran M (2005) Zeitgebers (time cues) for biological clocks. Curr Sci 89, 1136-1146.

[11] Berson DM, Dunn FA, Takao M (2002) Phototransduction by retinal ganglion cells that set the circadian clock. Science 295, 1070-1073.

[12] Provencio I, Rollag MD, Castrucci AM (2002) Photoreceptive net in the mammalian retina. Nature 415, 493-493.

[13] Chen S-K, Badea T, Hattar S (2011) Photoentrainment and pupillary light reflex are mediated by distinct populations of ipRGCs. Nature 476, 92-95.

[14] Kessel L, Siganos G, Jørgensen T, Larsen M (2011) Sleep disturbances are related to decreased transmission of blue light to the retina caused by lens yellowing. Sleep 34, 12151219.

[15] Turner PL, Mainster MA (2008) Circadian photoreception: Ageing and the eye's important role in systemic health. $\mathrm{Br}$ J Ophthalmol 92, 1439-1444.

[16] La Morgia C, Ross-Cisneros FN, Sadun AA, Carelli V (2017) Retinal ganglion cells and circadian rhythms in Alzheimer's disease, Parkinson's disease, and beyond. Front Neurol 8, 162.

[17] Van Erum J, Van Dam D, De Deyn PP (2018) Sleep and Alzheimer's disease: A pivotal role for the suprachiasmatic nucleus. Sleep Med Rev 40, 17-27.

[18] Wu Y-H, Swaab DF (2007) Disturbance and strategies for reactivation of the circadian rhythm system in aging and Alzheimer's disease. Sleep Med 8, 623-636.

[19] Falck RS, Davis JC, Best JR, Chan PC, Li LC, Wyrough AB, Bennett KJ, Backhouse D, Liu-Ambrose T (2020) Effect of a multimodal lifestyle intervention on sleep and cognitive function in older adults with probable mild cognitive impairment and poor sleep: A randomized clinical trial. $J$ Alzheimers Dis 76, 179-193.

[20] Hjetland GJ, Pallesen S, Thun E, Kolberg E, Nordhus IH, Flo E (2020) Light interventions and sleep, circadian, behavioral, and psychological disturbances in dementia: A systematic review of methods and outcomes. Sleep Med Rev 52, 101310.

[21] Shirani A, Louis EKS (2009) Illuminating rationale and uses for light therapy. J Clin Sleep Med 5, 155.

[22] Price LL, Lyachev A, Khazova M (2017) Optical performance characterization of light-logging actigraphy dosimeters. J Opt Soc Am A 34, 545-557.

[23] Lucas RJ, Peirson SN, Berson DM, Brown TM, Cooper HM, Czeisler CA, Figueiro MG, Gamlin PD, Lockley SW, O'Hagan JB (2014) Measuring and using light in the melanopsin age. Trends Neurosci 37, 1-9.

[24] International Commission on Illumination (2019) CIE position statement on non-visual effects of light: Recommending proper light at the proper time. $2^{\text {nd }}$ Edition.

[25] Price L, Khazova M, O'Hagan J (2012) Performance assessment of commercial circadian personal exposure devices. Light Res Technol 44, 17-26.

[26] Cao D, Barrionuevo PA (2015) Estimating photoreceptor excitations from spectral outputs of a personal light exposure measurement device. Chronobiol Int 32, 270-280.

[27] Figueiro M, Hamner R, Bierman A, Rea M (2013) Comparisons of three practical field devices used to measure personal light exposures and activity levels. Light Res Technol 45, 421-434.

[28] Shochat T, Umphress J, Israel AG, Ancoli-Israel S (1999) Insomnia in primary care patients. Sleep 22, S359-S365.

[29] Ancoli-Israel S, Klauber MR, Jones DW, Kripke DF, Martin J, Mason W, Pat-Horenczyk R, Fell R (1997) Variations in circadian rhythms of activity, sleep, and light exposure related to dementia in nursing-home patients. Sleep 20, 1823.

[30] Münch M, Wirz-Justice A, Brown SA, Kantermann T, Martiny $\mathrm{K}$, Stefani O, Vetter C, Wright Jr KP, Wulff K, Skene DJ (2020) The role of daylight for humans: Gaps in current knowledge. Clocks Sleep 2, 61-85.

[31] Ancoli-Israel S, Cole R, Alessi C, Chambers M, Moorcroft W, Pollak CP (2003) The role of actigraphy in the study of sleep and circadian rhythms. Sleep 26, 342-392.

[32] van Someren EJ, Mirmiran M, Swaab DF (1993) Nonpharmacological treatment of sleep and wake disturbances in aging and Alzheimer's disease: Chronobiological perspectives. Behav Brain Res 57, 235-253.

[33] Figueiro MG, Hamner R, Higgins P, Hornick T, Rea MS (2012) Field measurements of light exposures and circadian disruption in two populations of older adults. J Alzheimers Dis 31, 711-715.

[34] Ancoli-Israel S, Martin JL, Blackwell T, Buenaver L, Liu L, Meltzer LJ, Sadeh A, Spira AP, Taylor DJ (2015) The SBSM guide to actigraphy monitoring: Clinical and research applications. Behav Sleep Med 13, S4-S38.

[35] Bland JM, Altman D (1986) Statistical methods for assessing agreement between two methods of clinical measurement. Lancet 327, 307-310.

[36] Akobeng AK (2007) Understanding diagnostic tests 3: Receiver operating characteristic curves. Acta Paediatr 96, 644-647.

[37] Leibowitz HW, Owens DA (1991) Can normal outdoor activities be carried out during civil twilight? Appl Opt 30, 3501-3503.

[38] O'Caoimh R, Mannion H, Sezgin D, O'Donovan MR, Liew A, Molloy DW (2019) Non-pharmacological treatments for sleep disturbance in mild cognitive impairment and demen- 
tia: A systematic review and meta-analysis. Maturitas 127, 82-94.

[39] Morita T, Tokura H (1996) Effects of lights of different color temperature on the nocturnal changes in core temperature and melatonin in humans. Appl Human Sci 15, 243-246.
[40] Bierman A, Klein TR, Rea MS (2005) The Daysimeter: A device for measuring optical radiation as a stimulus for the human circadian system. Meas Sci Technol 16, 2292. 